\title{
Shape Identification for Distributed Parameter Systems and Temperature Profiles in Tokamaks
}

\author{
Emmanuel WITRANT ${ }^{1}$ and Sylvain BRÉMOND ${ }^{2}$
}

\begin{abstract}
Simple physical models are often difficult to obtain for nonhomogeneous transport phenomena that involve complex couplings between several distributed variables, such as temperature profiles in tokamak plasmas. Model-based current or combustion control approaches necessitate plasma models with real-time computation capabilities. This may prevent the use of classical knowledge-based physical models and motivates dedicated identification methods to exploit the large available experimental data. Based on distributed temperature measurements, this paper proposes a parameter-dependent identification method that first relates the normalized profiles distribution to a specific shape description thanks to a threehidden-layers neural network architecture. The amplitude of the profiles is then constrained by the global energy conservation (0D) with an identified time constant. The shape parameters and time constant are related to the global parameters using appropriate scaling laws. Experimental results illustrate the efficiency of the proposed identification method to estimate TORE SUPRA temperature profiles.
\end{abstract}

\section{INTRODUCTION}

Distributed parameter systems (DPS) constitute a class of systems which are particularly difficult to model accurately. They are often described by classical transport equations (i.e. convection-diffusion models) with time-varying coefficients and additional nonlinear terms. Their dynamics may strongly depend on the operating conditions and/or unmodeled coupling with other transport phenomena. Modeling is further complicated when space-dependent inputs are considered, which motivates the identification method proposed in this paper. Complex irrigation systems, car traffic, large antennas waveguides, fluids on surfaces or tokamak plasmas provide particularly challenging examples for such systems.

Most DPS identification methods rely on the spatial discretization of the partial differential equation (PDE) associated with the transport model to compute the convection and diffusion components when the system is affine (linear dependence) in the exogenous inputs. Orthogonal collocation [1], [2] and Galerkin's method [3] are generally used for this discretization. Identification of time-invariant linear DPS can be performed using orthogonal functions with the results proposed in [4], where some identifiability requirements are discussed. The case of parameter estimation for non-linear DPS is considered in [5] for systems with boundary inputs. The problem becomes more complicated for time-varying non-affine systems with distributed uncertain inputs, such as the tokamak plasmas.

The authors are with: 1 GIPSA-lab, Université Joseph Fourier / CNRS, BP 46, 38402 Saint Martin d'Hères, France; 2 CEA, IRFM, F-13108 Saint Paul-lez-Durance, France. E-mail: emmanuel.witrant@gipsa-lab.grenoble-inp.fr
Indeed, electron / ion temperature profiles in tokamak plasmas result from heat transfer processes for which reliable physical models are still not fully available. The global thermal energy dynamics is yet reasonably well represented by $0 \mathrm{D}$ energy confinement time scaling laws derived as linear regression on engineering quantities of existing tokamaks [6]. The 1D transfer (along the small tokamak radius) of the plasma heat through the so-called plasma equilibrium magnetic surfaces is often described using a diffusive-like non linear PDE with more or less ad-hoc diffusivity coefficients, as it is now recognized that transport of particles and heat within the tokamak plasmas is ruled by more complex turbulence processes. As a result, such a description provides a poor modeling benefit for a significant increase in complexity and computation time as far as control-oriented modeling, especially plasma current profile control, is concerned. Model-based control of the plasma current profile, a very active research area due to the key effect of this profile on both plasma stability and performance (see for example [7], [8], [9], [10] and references therein), yet requires some 1D modeling of the effect of tokamak actuators variables. This is especially relevant for the heating systems effect on plasma temperature, as the electrical resistivity and most noninductive current drive sources efficiencies strongly depend on the plasma temperature.

From an engineering point of view and for real-time model-based control purposes, we may then be more interested in establishing a simplified model that strongly relies on experimental measurements and guarantees the global physical consistency (such as OD mass or energy conservation) than obtaining a precise PDE model. This is the main motivation of the proposed identification method, where the normalized spatial distribution of the DPS is first reduced to shape parameters (identification of a sigmoid distribution in the plasma temperature case). These parameters are then related by scaling laws to global parameters (such as the plasma current, electrons density or toroidal magnetic field) that are easily known for some given operating conditions. Finally, the OD conservation dynamics is introduced by constraining the integral of the shape (corresponding to the total energy or mass of the system) to be consistent with the boundary inputs and outputs of the system, with an identified time constant.

The proposed approach can be compared with classical identification methods as follows. Instead of discretizing in space and identifying the parameter dependencies, the state is estimated with a set of parameter dependent (timevarying) functions that ensure the continuity and uniformity 
of the spatial distribution. This is achieved thanks to a spacedependent basis of functions that describes the DPS shape at each sampling time. The estimated model is then based on the identification of a finite set of time-varying shape parameters, which determines the dynamics of a sum of time and space dependent functions. This identification method is consequently well suited to model complex systems when distributed measurements are available and to capture the main input/output relationships for real-time applications (such as control-oriented models [11] or model-predictive control [12]).

This paper is organized as follows. The main hypotheses and a global description of the identification process are given in Section II. The spatial distribution, steady-state behavior and transient dynamics estimations are presented in Sections III to V, respectively. The efficiency of the proposed model is discussed in Section VI, thanks to experimental measurements of Tore Supra temperature profiles.

\section{Hypotheses and Method Overview}

The class of systems considered is DPS that involve transport phenomena, such as convection or diffusion. Such systems are generally modeled with nonlinear PDEs relating the dynamics of the distributed variable of interest $v(x, t)$ (the plasma temperature profile along the normalized small tokamak radius $x$ in our case) to a set of controlled engineering parameters or known disturbances $u(t)$ (such as the radio-frequency wave heating antenna power) thanks to an evolution law $\dot{v}=f(v, u, x, t)$ (this operator describes a transport phenomena with distributed and/or boundary inputs).

The following hypotheses are made on the transport phenomena and available signals:

H1) the transport phenomena is supposed to have smooth transitions $(f(\cdot)$ continuously differentiable);

H2) distributed measurements of $v(x, t)$ are available to identify the system (observable state);

$H 3)$ the exogenous inputs $u(t)$ are known;

$H 4)$ the normalized profiles $v(x, t) / v(0, t)$ exhibit some shape similarity that can be described by a functional basis (possibly non-linear) involving a limited number of time-varying parameters;

H5) stochastic disturbances are supposed to affect the system's input over a sufficient frequency range.

$H 1)$ excludes the shock waves from the analysis, as such transport phenomena would need a more dedicated identification approach. H2) is a reasonable assumption considering the fact that distributed measurement devices are available for large scale plants (such as Tokamaks). The need for distributed measurements may be removed if the system satisfies some traditional observability conditions. H3) restricts the use of global parameters to be known at each sampling time. $H 4$ ) is clearly the most restrictive hypothesis and will be discussed below. H5) relates to singular perturbation theory and is necessary to ensure that the signal is sufficiently rich to identify a relevant model.

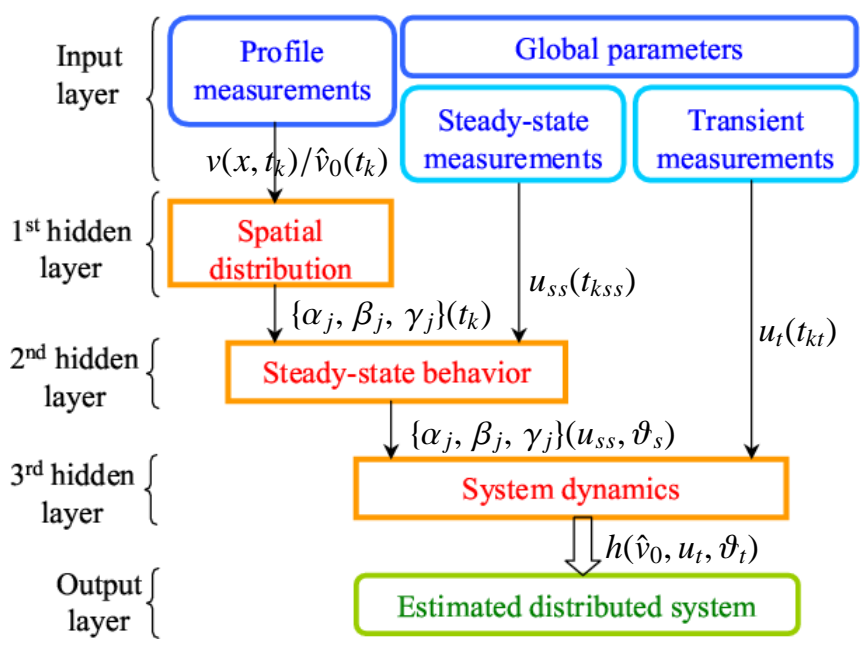

Fig. 1. Three hidden layer identification approach.

The proposed modeling approach relies on a shape description of the state profile. Instead of determining a lumped-parameter model at specific locations (as it is done with traditional finite elements techniques), we use the general nonlinear black-box structure (see [13] for a survey on nonlinear black-box modeling):

$$
\left.\frac{\hat{v}(x, t)}{\hat{v}_{0}(t)}=\sum_{j=1}^{n} \alpha_{j}(t) \kappa\left(\beta_{j}(t), \gamma_{j}(t), x\right)\right)
$$

where $\hat{v}(x, t)$ is the identified estimate of $v(x, t), \hat{v}_{0}(t)$ is the reference normalizing amplitude (i.e. $\hat{v}_{0}(t)=\hat{v}(0, t)$ in the example considered), and $\alpha_{j}(t), \beta_{j}(t)$ and $\gamma_{j}(t)$ are, respectively, the amplitude, slope and translation of the mother basis function that has a shape determined by $\kappa\left(\beta_{j}(t), \gamma_{j}(t), x\right)$ ) (i.e. a Gaussian or sigmoid distribution). The desired estimation precision is obtained by setting $n$ sufficiently large and the key issue is to choose, based on the visual observation of the measurements profiles, an appropriate class of functions $\kappa(\cdot)$ to ensures that the spatial distribution can be estimated with a minimum number of parameters. The advantage of this identification procedure is then limited by the ability to find a functional basis $\kappa$ that involve less parameters than traditional approaches (which relates to $H 4$ ).

The identification method is based on a three hidden layers network, presented on Fig. 1 (see [14] and related references for examples of distributed system identification using neural networks). The first two layers identify the shape parameters of the normalized profile $\hat{v}(x, t) / \hat{v}_{0}(t)$, while the dynamics is introduced in the third layer along with $\dot{\hat{v}}_{0}(t)$. We distinguish here the steady state behavior, which corresponds to small variations around an equilibrium and almost constant inputs $u_{s s}\left(t_{k s s}\right)$, from the transient response, where we can observe a transition in the operation mode and large variations of the inputs $u_{t}\left(t_{k t}\right)$. This is mainly motivated by the fact that we identify algebraic relationships in the first case while a dynamic algorithm is needed in the second case. Note also that the global behavior of the system can be described with low sampling rate data measured on large time intervals when 
the system has slow variations while the transient behavior needs a high sampling rate and measurements focused on the transition phases.

The time vector associated with the distributed measurements (including both $t_{k s s}$ and $t_{k t}$ ) is denoted as $t_{k}$ and the parameters computed by each layer are $\left\{\alpha_{j}, \beta_{j}, \gamma_{j}\right\}\left(t_{k}\right), \vartheta_{s}$ and $\vartheta_{t}$. The three layers of the network are then described as follows:

1) the first layer is focused on the spatial variation: for each sampling time $t_{k}$, it estimates $\hat{v}\left(x, t_{k}\right) / v\left(0, t_{k}\right)$ from the normalized measurements $v\left(x, t_{k}\right) / v\left(0, t_{k}\right)$ and provides the shape parameters $\left\{\alpha_{j}, \beta_{j}, \gamma_{j}\right\}\left(t_{k}\right)$ (outputs) according to (1) for the next layer;

2) the second layer is focused on the steady-state behavior and replaces the time-dependency of $\left\{\alpha_{j}, \beta_{j}, \gamma_{j}\right\}$ by scaling laws involving the time-dependent global parameters $u_{s s}\left(t_{k}\right)$ and constant identified parameters $\vartheta_{s}$, thus expressing the shape parameters in terms of $u_{s s}$ and $\vartheta_{s}$ (denoted as $\left\{\alpha_{j}, \beta_{j}, \gamma_{j}\right\}\left(u_{s s}, \vartheta_{s}\right)$ on Fig. 1;

3) finally, the transient dynamics is considered in the third layer where the time-variation law :

$$
\frac{d \hat{v}_{0}}{d t}=h\left(\hat{v}_{0}, u_{t}, \vartheta_{t}\right), \hat{v}_{0}(0)=\hat{v}_{0 i}
$$

is introduced to model $v\left(0, t_{k}\right)$ thanks to a specific choice of $h(\cdot)$ and the identified parameters $\vartheta_{t}$.

The specific choice of $\kappa(\cdot)$ is based on the general shape of $v(\cdot)$ while $h(\cdot)$ can be set from the physical properties of the system (such as a conservation law). A stochastic gradient method ensures the optimal parameter identification (least squares minimization) and allows for the consideration of parameter dependencies and nonlinear aspects on each layer, as detailed in the next sections. More details on the sensitivity-based gradient computation used in this paper and the consideration of the system dynamics are provided in [15].

\section{Shape Definition of the Spatial Distribution}

The first step is to approximate the measured distributed state with a finite number of parameter-dependent continuous functions. More precisely, given the spatial distribution $v\left(x, t_{k}\right)$ and a set of trial functions, we want to determine the optimal set of parameters $\vartheta_{f}\left(t_{k}\right) \doteq\left\{\alpha_{i}, \beta_{i}, \gamma_{i}\right\}$ in (1) which minimizes the variance of $v\left(x, t_{k}\right)-\hat{v}\left(x, t_{k}\right)$, where $\hat{v}$ is the model output. This is done by minimizing the quadratic cost:

$$
J_{f}\left(t_{k}\right)=\sum_{j=1}^{N_{x}}\left[\frac{v\left(x_{j}, t_{k}\right)-\hat{v}\left(x_{j}, t_{k}\right)}{v\left(0, t_{k}\right)}\right]^{2}
$$

where $N_{x}$ is the number of measurement locations.

Remark 1: If a specific class of associated transport model is available (e.g. with a partial differential equation with constant coefficients), such knowledge can be included in the shape definition. In this case, the (quasi)steady-state solution of the PDE can be used to define the mother function support.

\section{Steady-State behavior}

The steady-state behavior of the identified system is modeled in the second layer by establishing the relationship between the spatial parameters $\vartheta_{f}\left(t_{k s s}\right)$ (at time instants when the system is at equilibrium) and some global, OD (only timevarying) inputs $u_{i} \in \mathbb{R}^{n_{i}}$. The spatial parameters determined by the first layer then provide for the reference outputs of the second layer.

\section{A. Linear regression}

Parameter estimation methods are particularly efficient when the output is estimated with a linear regression technique (guaranteed global minimum), which can be obtained from an exponential scaling law as follows. In this case $\hat{\vartheta}_{f}\left(t_{k s s}\right)$, the estimate of the parameters given by the first layer, writes as:

$$
\vartheta_{f} \approx\left\{\begin{array}{c}
\hat{\vartheta}_{f, 1}(t)=e^{\vartheta_{s, 0,1}} u_{1}^{\vartheta_{s, 1,1}} u_{2}^{\vartheta_{s, 2,1}} u_{3}^{\vartheta_{s, 3,1}} \ldots u_{n_{i}}^{\vartheta_{s, n_{i}, 1}} \\
\vdots \\
\hat{\vartheta}_{f, 3 n}(t)=e^{\vartheta_{s, 0,3 n}} u_{1}^{\vartheta_{s, 1,3 n}} u_{2}^{\vartheta_{s, 2,3 n}} u_{3}^{\vartheta_{s, 3,3 n}} \ldots u_{n_{i}}^{\vartheta_{s, n}, 3 n}
\end{array}\right.
$$

where the time dependency of $u_{s s} \doteq\left\{u_{1}, u_{2}, \ldots, u_{n i}\right\}$ in $t_{k s s}$ is omitted to simplify the notations. This set of equations writes in the linear form:

$$
\ln \left(\hat{\vartheta}_{f}\right)=\left[1 \ln \left(u_{s s}\right)\right] \times \vartheta_{s}
$$

with:

$$
\vartheta_{s} \doteq\left[\begin{array}{ccc}
\vartheta_{s, 0,1} & \ldots & \vartheta_{s, n_{i}, 1} \\
\vdots & \ddots & \vdots \\
\vartheta_{s, 0,3 n} & \ldots & \vartheta_{s, n_{i}, 3 n}
\end{array}\right] \in \mathbb{R}^{3 n \times\left(n_{i}+1\right)}
$$

and $\vartheta_{s}$ minimizes the cost function:

$$
J_{s}\left(\vartheta_{s}\right)=\frac{1}{n_{m}} \sum_{i=1}^{n_{m}}\left\|\ln \left(\vartheta_{f}(i)\right)-\ln \left(\hat{\vartheta}_{f}(i)\right)\right\|^{2}
$$

\section{B. Nonlinear / discontinuous dependencies}

The more general case where $\vartheta_{f}$ is estimated with a nonlinear function does not introduce any particular technical difficulty as long as $\hat{\vartheta}_{f} \in C^{1}$, where $C^{1}$ is the set of continuously differentiable functions (the stochastic gradient descent method applies). A more interesting case is when a specific triggering input $u_{T}$ introduces some discontinuities in the model considered. In this case, an appropriate identification scheme has to include a possible switching between different submodels. This kind of behavior can be observed when $u_{T}$ reflects an actuator switching, some particular microscopic phenomena or unmodeled instabilities.

We consider that each submodel is independent from the others, and consequently that the input data corresponding to submodel $i$ does not influence the optimal parameter set of submodel $j$, with $j \neq i$. The second hidden layer then writes in the switched form as:

$$
\hat{\vartheta}_{f}\left(t_{k s s}\right)=\left\{\begin{array}{cll}
g^{1}\left(u_{s s}, \vartheta_{s}^{1}\right), & \text { if } & u_{T}\left(t_{k s s}\right) \in\left[a_{1}, a_{2}\right] \\
\vdots & & \vdots \\
g^{N}\left(u_{s s}, \vartheta_{s}^{N}\right), & \text { if } & u_{T}\left(t_{k s s}\right) \in\left[a_{N}, a_{N+1}\right]
\end{array}\right.
$$


where $g^{i}(\cdot) \in C^{1}$ is the fitting law corresponding to the specific value of the triggering input, $i$ indicates the model considered and $\left[a_{i}, a_{i+1}\right]$ the triggering interval. The optimal set of parameters $\vartheta_{s}$ is now a set of $N$ matrices, obtained for example with a gradient descent method.

\section{Amplitude Dynamics}

The third hidden layer focuses on the dynamics associated with the transient response of the system. The function $h(\cdot)$ has to be such that $d \hat{v}_{0} / d t \rightarrow 0$ when the selected inputs $u_{t}$ are constant and vary otherwise. Supposing that the inputs $u_{s s}$ and $u_{t}$ have faster dynamics than $\hat{v}_{0}$ (i.e. the actuators and sensors dynamics do not interfere in the identification), the previous property is verified if we restrict $h(\cdot)$ to the class of functions that writes as $h\left(\phi\left(u_{t}, \vartheta_{t}\right)-\hat{v}_{0}, u_{t}, \vartheta_{t}\right)$ where $\phi\left(u_{t}, \vartheta_{t}\right)$ is the equilibrium value of $\hat{v}_{0}$. It is supposed that:

- $\phi(\cdot)$ is a $C^{1}$ function;

- $h(\cdot)$ is Lipschitz continuous and strictly decreasing in $\hat{v}_{0}$, to ensure that $d \hat{v}_{0} / d t \rightarrow 0$ for constant inputs as well as the convergence of the ODE associated with the sensitivity computation.

The state $\hat{v}_{0}$ then converges to $\phi\left(u_{t}, \vartheta_{t}\right)$ and the optimal set of parameters $\vartheta_{t}^{*}$ is obtained using a gradient descent method with sensitivity $S\left(\vartheta_{t}, t\right)=\partial \hat{v}_{0} / \partial \vartheta_{t}$ where $\partial \hat{v}_{0} / \partial \vartheta_{t}$ is computed by solving on $[0, T]$ the ODE:

$$
\frac{d}{d t}\left[\frac{\partial \hat{v}_{0}}{\partial \vartheta_{t}}\right]=-\frac{\partial h}{\partial\left(\phi-\hat{v}_{0}\right)} \frac{\partial \hat{v}_{0}}{\partial \vartheta_{t}}+\frac{\partial h}{\partial \vartheta_{t}}
$$

along with (2).

\section{Experimental Results}

The proposed identification method is applied to the estimation of the electron temperature profile $T_{e}(x, t)$ of Tore Supra tokamak plasmas operating in L-mode. The temperature behavior is generally set by the diffusion equation

$$
\frac{3}{2} \frac{\partial n_{e} T_{e}}{\partial t}=\nabla\left(n_{e} \chi_{e} \nabla T_{e}\right)+S_{T}
$$

where $n_{e}(x, t)$ is the electron density, $\chi_{e}(x, t)$ is the temperature diffusivity and $S_{T}(x, t)$ corresponds to distributed heat sources (radio frequency antennas). Various approaches have been proposed for the computation of $\chi$ and $S_{T}$ but their number illustrates the difficulty to model the heat diffusion for tokamak plasmas. Some existing fitting laws provide for the volume average temperature estimation [6] but do not allow to estimate the spatial distribution, which motivated the proposed approach.

The first and second layer of the neural network are used to determine the profile shape while the third layer sets its amplitude. The considered system inputs $u(t)$ are global parameters, which are usually set before a shot (tokamak plasma experiment) and can be used in predictive control schemes. A qualitative analysis of the plasma physics motivates the choice of the following global variables:

$$
u(t) \doteq\left\{I_{p}, B_{\phi_{0}}, \bar{n}_{e}, P_{t o t}, P_{l h}, N_{/ /}, P_{i c r f}\right\}
$$

(in MA, T, $10^{19} \mathrm{~m}^{-3}, \mathrm{MW}$ ), where $I_{p}$ is the total plasma current, $B_{\phi_{0}}$ is the toroidal magnetic field, $\bar{n}_{e}$ is the electron line average density and $P_{t o t}$ is the total power input. The second part of the input data comes from the distributed sources with the power $P_{l h}$ and parallel refraction index $N_{/ /}$ of the Lower Hybrid launchers and the power of the Ion Cyclotron Radio Frequency antenna $P_{\text {icrf }}$.

\section{A. Profile shape estimation}

The profiles shape (first layer) is estimated using the normalized measured temperature profiles $T_{e}(x, t) / T_{e 0}(t)$, where $T_{e 0}(t) \doteq T_{e}(0, t)$ is the central temperature and $x$ is the normalized small plasma radius. The spatial distribution is estimated with the sigmoid distribution:

$$
\frac{T_{e}(x, t)}{T_{e}(0, t)} \approx \frac{\hat{v}(x, t)}{\hat{v}_{0}(t)}=\frac{\alpha}{1+e^{-\beta(x-\gamma)}}
$$

Note that, considering the measurements precision and the $L$-mode operation, a single sigmoid function is sufficient to obtain the desired accuracy. Additional sigmoids would be needed to represent the $H$-mode or internal transport barriers. The set of first layer optimal parameters $\vartheta_{f}\left(t_{k}\right)=\{\alpha, \beta, \gamma\}$ is obtained by minimizing the cost function:

$$
J_{f}\left(t_{k}\right)=\sum_{j=1}^{N_{x}} \epsilon_{f}\left(x_{j}, t_{k}\right)^{2}, \quad \epsilon_{f}\left(x_{j}, t_{k}\right)=\left|\frac{T_{e}\left(x_{j}, t_{k}\right)-\hat{v}\left(x_{j}, t_{k}\right)}{T_{e 0}\left(t_{k}\right)}\right|
$$

where $N_{x}=21$ is the number of spatial measurements available to set the identification method and $t_{k}=1 \ldots N_{t}$. The resulting optimal values of the cost function $J_{f}^{*}$ at each $t_{k}$ are presented at the top of Figure 2, where the 19 shots are plotted consecutively on the same time scale and delimited with vertical dotted lines. The stars and the vertical dotted lines indicate the times for switching between shots (lower stars) and between phases with different operating conditions (top stars). All the shots considered have an associated $J_{f}^{*}<10^{-3}$ (the algorithm is stopped when $J_{f}<10^{-4}$ to reduce the amount of computations), which validates the fact that a single sigmoid function provides a good approximation for the temperature profile shape.

\section{B. Parameter dependency}

The second layer is estimated combining the linear regression proposed in Section IV-A and the switched model described in Section IV-B, where the switching term is $P_{l h}$ (optimization tests highlighted the peculiar influence of this parameter). The data set for $\hat{v}\left(x, t_{k s s}\right) / \hat{v}_{0}\left(t_{k s s}\right)$ and $u\left(t_{k s s}\right)$ is chosen such that $u$ has slow variations compared to the temperature dynamics.

The switched scaling law for the shape parameters writes as:

$$
\hat{\vartheta}_{f}=\left\{\begin{array}{l}
\left\{\alpha_{l h}, \beta_{l h}, \gamma_{l h}\right\} \quad \text { if } \quad P_{l h} \neq 0 \\
\left\{\alpha_{\omega}, \beta_{\omega}, \gamma_{\omega}\right\} \quad \text { else. }
\end{array}\right.
$$

with

$$
\left\{\begin{array}{l}
\alpha_{l h}=e^{-0.87} I_{p}^{-0.43} B_{\phi_{0}}^{0.63} N_{/ /}^{0.25}\left(1+\frac{P_{\text {icrf }}}{P_{\text {tot }}}\right)^{0.15} \\
\beta_{l h}=-e^{3.88} I_{p}^{0.31} B_{\phi_{0}}^{-0.86} \bar{n}_{e}^{-0.39} N_{/ /}^{-1.15} \\
\gamma_{l h}=e^{1.77} I_{p}^{1.40} B_{\phi_{0}}^{-1.76} N_{/ /}^{-0.45}\left(1+\frac{P_{\text {icrf }}}{P_{\text {tot }}}\right)^{-0.54}
\end{array}\right.
$$



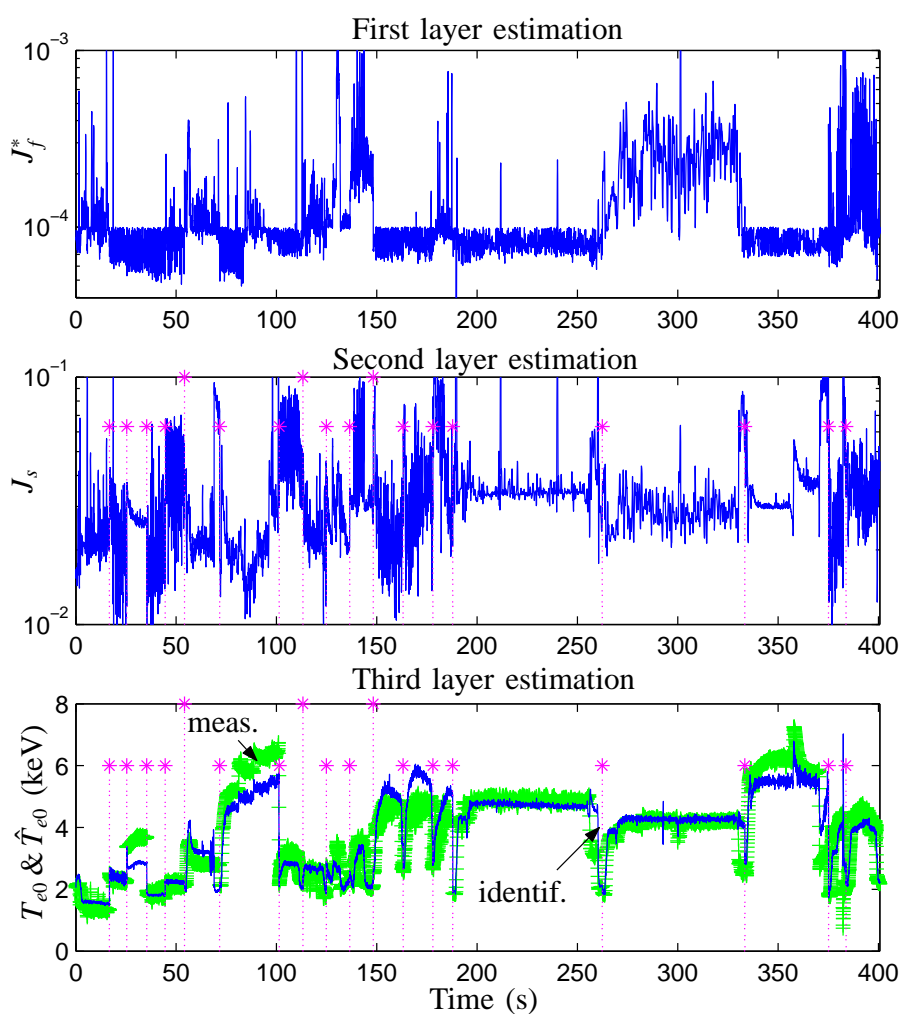

Fig. 2. Identification method efficiency when applied to the reference data set (19 shots, 9500 measurements).

$$
\left\{\begin{array}{l}
\alpha_{\omega}=e^{-0.37} I_{p}^{-0.46} B_{\phi_{0}}^{0.23} \bar{n}_{e}^{0.22} \\
\beta_{\omega}=-e^{1.92} I_{p}^{0.38} \bar{n}_{e}^{-0.33} \\
\gamma_{\omega}=e^{-0.15} I_{p}^{1.03} B_{\phi_{0}}^{-0.51}\left(1+\frac{P_{\text {icrf }}}{P_{\text {tot }}}\right)^{-0.46}
\end{array}\right.
$$

Note that only the most significant dependencies are kept in the final scaling laws. For example, it appeared that $P_{l h}$ has a limited impact on the profile slope $\left(\beta_{l h}\right)$ while it significantly affects the other two parameters $\left(\alpha_{l h}\right.$ and $\left.\gamma_{l h}\right)$. The ratio $P_{\text {icrf }} / P_{\text {tot }}$ is used to take into account the fact that the ICRF antenna significantly modifies the shapes when it is used but does not necessitate the definition of an extra switch.

This model is compared with experimental data on the middle part of Figure 2. The minimized cost $J_{s}$ is computed as $J_{f}$ but with $\hat{v}\left(x_{k}, t_{k s s}\right) / \hat{v}_{0}\left(t_{k s s}\right)$ estimated using (7)-(8). The average value of the cost function is increased but remains within some acceptable bounds with a mean value of $3.5 \%$. This, along with the fact that the distribution of the error around its mean value doesn't exhibit significantly large shot-dependent variations, validates the specific choice of $\left\{I_{p}, B_{\phi_{0}}, \bar{n}_{e}, P_{l h}, N_{/ /}, P_{i c r f}\right\}$ as system inputs and the linear regression approach.

\section{System dynamics}

The third layer estimates the dynamics of the central temperature $T_{e 0}(t)$ as $h(\cdot)$, including an energy conservation constraint. Supposing that the tokamak torus shape can be approximated with a cylinder (classical cylindrical approximation [16], [11]), the physics of plasma energy confinement is introduced to relate $T_{e 0}(t)$ to the plasma energy:

$$
W(t)=\frac{3 e}{2} \int_{V}\left(n_{e} T_{e}+n_{i} T_{i}\right) d V \approx C \int_{0}^{1}\left(n_{e} T_{e}+n_{i} T_{i}\right) d x
$$

where $C \doteq 6 \pi^{2} a^{2} R_{0} e$ with $a$ and $R_{0}$ the minor and major plasma radius, respectively, and $e$ the electron charge. The subscript $i$ denotes the ions contribution. The ratio $\alpha_{T i}(t) \doteq$ $T_{i}(0, t) / T_{e}(0, t)$ is estimated using the linear regression technique (on a set of 34 shots) as:

$\alpha_{T i}(t)=1-0.31\left(\frac{I_{p}}{B_{\phi_{0}}}\right)^{-0.38} \bar{n}_{e}^{-0.90}\left(1+\frac{P_{i c r f}}{P_{t o t}}\right)^{-1.62}\left(1+\frac{P_{l h}}{P_{t o t}}\right)^{1.36}$

and the electronic density is approximated with $n_{e}(x, t) \approx$ $\left(1-x^{\gamma_{n}}\right) n_{e}(0, t)$, where $\gamma_{n}=2$. Approximating the ratio of ion to electron density with $\alpha_{n i}(t) \approx\left(7-Z_{e f f}(t)\right) / 6$, where $Z_{\text {eff }}(t)$ is the effective plasma charge, the energy equation implies that $T_{e 0}(t)=\mathcal{A}(t) W(t)$ with:

$$
\mathcal{A}(t) \doteq\left[C\left(1+\alpha_{T i} \alpha_{n i}\right) \frac{\gamma_{n}+1}{\gamma_{n}} \bar{n}_{e} \int_{0}^{1}\left(1-x^{\gamma_{n}}\right) x \frac{\hat{v}\left(x_{k}, t\right)}{\hat{v}_{0}(t)} d x\right]^{-1}
$$

The relationship between $W$ and $P_{\text {tot }}$ corresponds to first order dynamics and the optimal parameters $\left\{\vartheta_{t 0} \ldots \vartheta_{t 4}\right\}$ set a scaling law on the time constant to minimize:

$$
J_{t}=\frac{1}{N_{t}} \sum_{i=1}^{N_{t}}\left(T_{e 0}(i)-\hat{T}_{e 0}(i)\right)^{2} d t(i)
$$

More precisely, the estimated central temperature $\hat{T}_{e 0}(t)$ results from the proper approximation of the thermal confinement time $\tau_{t h}(t)$, which sets the dynamics:

$$
\left\{\begin{aligned}
\tau_{t h} & =0.14 I_{p}^{0.91} B_{\phi_{0}}^{-0.13} \bar{n}_{e}^{0.77} P_{t o t}^{-0.75} \\
\frac{d W}{d t} & =P_{t o t}-\frac{1}{\tau_{t h}} W, \quad W(0)=P_{t o t}(0) \tau_{t h}(0) \\
\hat{T}_{e 0}(t) & =\mathcal{A} W
\end{aligned}\right.
$$

The efficiency of this layer, which also includes the results of the first and second layers since the integral of $\hat{v}(x, t) / \hat{v}_{0}(t)$ is needed to compute $\mathcal{A}$, is illustrated at the bottom of Figure 2, where both $T_{e 0}(t)$ and $\hat{T}_{e 0}(t)$ are presented. The accuracy of the estimation validates the use of (9) to estimate the central temperature as well as the efficiency of the proposed methodology to find the optimal parameters.

\section{Validation on a shot not included in the database}

To conclude on the experimental validation, the identified model is tested on a shot that is not included in the database (35557) on Figure 3. The first and last $2 s$ correspond to the current ramp-up and ramp-down, where some specific phenomena (not considered in the proposed model) occur. The distributed error $\left|T_{e}(x, t)-\hat{v}(x, t)\right|$ is presented on the top part of the figure and illustrates the spatial distribution accuracy. The highest error (approximately 10\%) appears when the sole input is the LH power but remains within acceptable bounds for the intended use. This effect is attenuated when LH is combined with ICRF and a more precise model could be obtained by distinguishing the case when LH is the only input from the case when both LH 


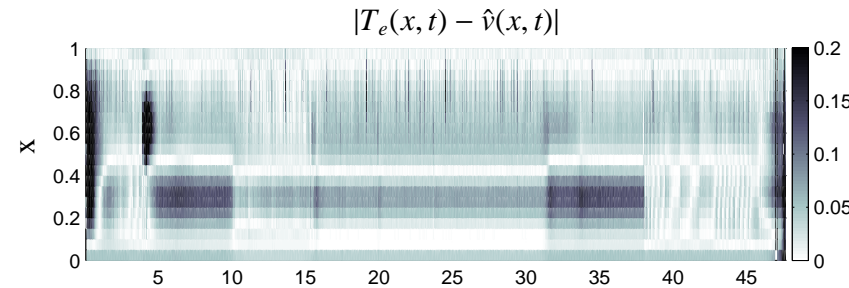

Central temperature $(\mathrm{keV})$ and power inputs $(\mathrm{MW})$

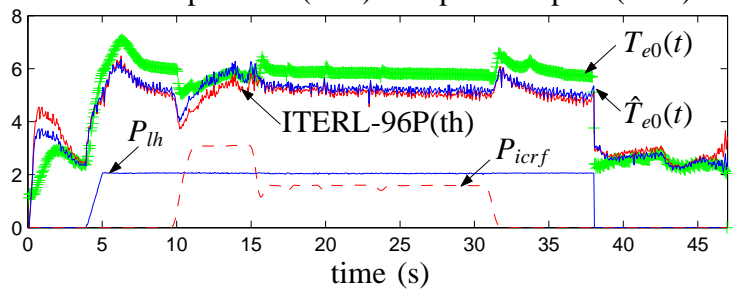

Fig. 3. Comparison of the model with a shot not included in the database (TS 35 557: $I_{p}=0.6 \mathrm{MA}, B_{\phi 0}=3.53-3.59 \mathrm{~T}, \bar{n}_{e}=1.4-2.6 \times 10^{19} \mathrm{~m}^{-3}$, $N_{/ /}=1.84$ and $Z_{\text {eff }}=2-5.5$ ).

and ICRF are used or by refining the shape description (i.e. adding a Gaussian shape, as suggested in [11]). The central temperature obtained from the measurements, from the proposed identification method and from the fitting law ITERL-96P(th) [6] $\tau_{\text {th }, \text { ITER }}=0.14 I_{p}^{0.96} B_{\phi_{0}}^{0.03} \bar{n}_{e}^{0.40} P_{\text {tot }}^{-0.73}$ are presented on the bottom part of the figure, along with $P_{l h}$ and $P_{\text {icrf }}$. The overall temperature profile is estimated with a satisfactory accuracy and is comparable with ITERL-96P(th) to estimate the plasma confinement. Further validation was given in [11], where this model was used for magnetic flux and current profiles prediction.

\section{Conclusions}

The problem of modeling non-homogeneous transport phenomena is considered in this work as defining a set of time and space varying functions that characterizes the shape distribution and its evolution. This is an alternative to classical discretization methods and ensures the spatial continuity of the resulting model. It also allows to set scaling laws with a direct interpretation of the physical phenomena. Based on some distributed measurements, the proposed identification method first estimates the spatial distribution at each sampling time with a sum of spacedependent functions. The steady-state and transient behaviors of the system, including the parameters dependencies, are then considered successively. The generality of the results allows to consider a large class of physical systems and to include a priori knowledge of the system explicitly in the choice of key global parameters. Experimental results illustrate the efficiency of this method for Tore Supra plasmas temperature profiles estimation.

\section{ACKNOWLEDGMENTS}

This work was carried out within the framework of the European Fusion Development Agreement and the French Research Federation for Fusion Studies. It is supported by the European Communities under the contract of Association between EURATOM and CEA. The views and opinions expressed herein do not necessarily reflect those of the European Commission. The research leading to these results has also received funding from the European Union Seventh Framework Programme [FP7/2007-2013] under grant agreement $\mathrm{n}^{o} 257462$ HYCON2 Network of excellence.

\section{REFERENCES}

[1] B. Finlayson, The method of weighted residuals and variational principles. New York: Academic Press, 1972.

[2] J. Funkquist, "Grey-box identification of a continuous digester - a distributed-parameter process," Control Engineering Practice, vol. 5, no. 7, pp. 919-930, July 1997.

[3] M. Polis, R. Goodson, and M. Wozny, "On parameter identification for distributed systems using galerkin's criterion," Automatica, vol. 9, no. 1, pp. 53-64, 1973.

[4] B. M. Mohan and K. B. Datta, "Linear time-invariant distributed parameter system identification via orthogonal functions," Automatica, vol. 27, no. 2, pp. 409-412, Mar. 1991.

[5] Y. Sunahara, S. Aihara, and F. Kojima, "A method for parameter estimation of a class of non-linear distributed systems under noisy observations," Automatica, vol. 22, no. 6, pp. 727-732, Nov. 1986.

[6] ITER Physics Expert Groups, "Chapter 2: Plasma confinement and transport," Nuclear Fusion, vol. 39, no. 12, pp. 2175-2249, 1999.

[7] D. Moreau et al., "A two-time-scale dynamic-model approach for magnetic and kinetic profile control in advanced tokamak scenarios on jet," Nuclear Fusion, vol. 48, no. 10, p. 106001, 2008. [Online]. Available: http://stacks.iop.org/0029-5515/48/i=10/a=106001

[8] F. Bribiesca Argomedo, C. Prieur, E. Witrant, and S. Brémond, "Polytopic control of the magnetic flux profile in a tokamak plasma," in 18th IFAC World Congress, Milan, Italy, Aug. 2011.

[9] A. Gahlawat, M. Peet, and E. Witrant, "Control and verification of the safety-factor profile in tokamaks using sum-of-squares polynomials," in 18th IFAC World Congress, Milan, Italy, Aug. 2011.

[10] Y. Ou, C. Xu, E. Schuster, T. Luce, J. Ferron, M. Walker, and D. Humphreys, "Optimal tracking control of current profile in tokamaks," IEEE Transactions on Control Systems Technology, 2011.

[11] E. Witrant, E. Joffrin, S. Brémond, G. Giruzzi, D. Mazon, O. Barana, and P. Moreau, "A control-oriented model of the current control profile in tokamak plasma," Plasma Phys. Control. Fusion, vol. 49, pp. 1075$1105,2007$.

[12] H. Ouarit, S. Brémond, R. Nouailletas, J.-F. Artaud, V. Basiuk, E. Witrant, and L. Autrique, "Model based predictive control of tokamak plasma current profile," in 26th Symposium on Fusion Technology (SOFT), Porto, Portugal, Sept. 2010.

[13] J. Sjöberg, Q. Zhang, L. Ljung, A. Benveniste, B. Delyon, P.-Y Glorennec, H. Hjalmarsson, and A. Juditsky, "Nonlinear black-box modeling in system identification: a unified overview," Automatica, vol. 31, no. 12, pp. 1691-1724, Dec. 1995.

[14] I. Arsie, C. Pianes, and M. Sorrentino, "A procedure to enhance identification of recurrent neural networks for simulating airfuel ratio dynamics in SI engines," Engineering Applications of Artificial Intelligence, vol. 19, no. 1, pp. 65-77, Feb. 2006.

[15] E. Witrant, D. Georges, and C. Canudas de Wit, "Optimal control design for the stabilization of network controlled systems," in Proc. of the IEEE American Control Conference, Minneapolis, MN (USA), June 2006.

[16] J. Blum, Numerical Simulation and Optimal Control in Plasma Physics, ser. Wiley/Gauthier-Villars Series in Modern Applied Mathematics. Gauthier-Villars, John Wiley \& Sons, 1989. 